\title{
DEVELOPMENT OF DYNAMIC STIFFNESS METHOD FOR FREE VIBRATION OF FUNCTIONALLY GRADED TIMOSHENKO BEAMS
}

\author{
H. Su ${ }^{\mathrm{a}}$ and J.R. Banerjee ${ }^{\mathrm{b} *}$ \\ a School of Science and Technology, University of Northampton, \\ Northampton, NN2 6JD, UK \\ ${ }^{\mathrm{b}}$ School of Engineering and Mathematical Sciences, City University London, \\ Northampton Square, London EC1V 0HB, UK
}

\begin{abstract}
The free vibration of functionally graded Timoshenko beams is investigated by developing the dynamic stiffness method. Material properties of the beam are assumed to vary continuously in the thickness direction. The governing differential equations of motion are solved and expressions for axial force, shear force and bending moment are derived. The dynamic stiffness matrix is then formulated by relating the amplitudes of forces and displacements at the ends of the beam. The Wittrick-Williams algorithm is used as solution technique to yield the natural frequencies and mode shapes of some illustrative examples. The results are discussed and some conclusions are drawn.
\end{abstract}

Keywords: Free vibration, functionally graded beams, dynamic stiffness method, Wittrick-Williams algorithm, Timoshenko beam theory.

* Corresponding author Email: j.r.banerjee@city.ac.uk

Tel: +44 (0)20 70408924, Fax: +44 (0)2070408566 


\section{Introduction}

Functionally graded materials (FGM) have continuous transition of material properties as a function of position along certain directions and thus are regarded as most promising applications of advanced composite materials as opposed to traditional isotropic and homogeneous materials. The gradual variation of material properties can be tailored to suit specific purposes in engineering design. Design of aircraft and space vehicles structures, electronic and biomedical installations are some examples where FGM can be fruitfully exploited. It is important to understand the static and dynamic behaviour of structural components made from FGM which has attracted many researchers in recent years particularly for beam structures that are widely used in aeronautical, civil, mechanical and other installations. As a consequence, the dynamic behaviour of functionally graded beams (FGBs) has become a fertile area of research and the literature is steadfastly growing [1-23]. A brief review of this carefully selected sample of literature is summarised below.

Alshorbagy et al. [1] and Chakraborty et al. [4] used finite element method (FEM) to investigate the free vibration characteristics of FGBs whereas Aydogdu and Taskin [2], Li [10], Librescu et al. [11], Lu and Chen [13], Oh et al. [14], Simsek [16], Sina et al. [17], Thai and Vo [18] and Xiang and Yang [20] used direct analytical approach to solve the problem. Giunta et al. [5] addressed the investigation somehow differently by applying axiomatic hierarchical theories through variational formulation to derive the governing differential equations and associated boundary conditions. Lai et al. [9] used a perturbation technique to deal with the large amplitude vibration of FGBs and in particular, studied the effects of boundary 
conditions on non-linear frequencies. Researchers who relied on elasticity solution to solve the static problems of FGBs as opposed to the dynamic ones include Birsan et al. [3] and Sankar [15] and Zhong and Yu [21]. By contrast Loja et al. [12] used a set of FEM models based on first and higher order shear deformation theories to investigate sandwich functionally graded particulate composites. Application of other approaches such as the Fouries series and Galerkin methods can be found in the work of Zhu and Sanker [22]. There are relatively very few authors who have carried out experiments to verify theoretical predictions of free vibration characteristics of FGBs, see for examples, Kapuria et al. [8] and Wattanasakulpong et al. [19]. For free vibration analysis of non-uniform FGBs, interested readers are referred to the works of Huang and Li [6] and Huang et al. [7].

Apparently, there has been very little effort to solve the free vibration problem of FGBs using the dynamic stiffness method (DSM). The current research is based on earlier research $[23,24]$ using DSM, but includes many additional features with wide-ranging results for free vibration of FGBs. It focuses on applying the DSM when investigating the free vibration behaviour of FGBs for different boundary conditions. The DSM uses exact member theory based on frequency dependent shape functions obtained from the exact solution of the governing differential equations of motion in free vibration. The method provides exact results for all natural frequencies and mode shapes without making any approximation en route. The DSM is recognizably the most accurate method in free vibration analysis, significantly superior to traditional finite element and other approximate methods. 
In the current investigation the material properties of the FGB are chosen to vary continuously through the beam thickness direction according to a power law distribution. The kinetic and potential energies are formulated using the first-order shear deformation theory generally known as Timoshenko beam theory (TBT) which accounts for the effects of shear deformation and rotary inertia. The governing differential equations of motion in free vibration are derived using Hamilton's principle and making use of symbolic computation [25]. The analytical expressions for axial force, shear force and bending moment at any cross-section of the FGB are obtained as a by-product of the Hamiltonian formulation. For harmonic oscillation, the governing differential equations are solved in closed analytical form for axial displacement, bending displacement and bending rotation. The boundary conditions for displacements and forces are imposed in algebraic form to derive the dynamic stiffness matrix of the FGB by relating the amplitudes of the forces to those of the displacements at the ends of the beam. Once the dynamic stiffness matrix of the FGB is developed, the eigenvalue problem is solved by means of the Wittrick and Williams algorithm [26] yielding natural frequencies and mode shapes of the FGB. The investigation required a substantial amount of validation exercise in that results obtained from the present theory are compared with the ones available in the literature. Next, a parametric study is carried out by varying significant beam parameters, the power law index, the length to thickness ratio and boundary conditions of the beam. Numerical results are discussed and this is followed by some concluding remarks. 


\section{Theory}

\subsection{Derivation of the governing differential equations}

A uniform FGB with a rectangular cross section in a right-handed Cartesian coordinate system is shown in Fig. 1. The beam has a length $L$, width $b$, and thickness $h$. Material properties of the beam are Young's modulus $E$, Poisson's ratio $v$, shear modulus $G$, and mass density $\rho$. It is assumed that the effective material properties $P(\mathrm{z})$, satisfying all the material properties, vary continuously in the thickness direction $(\mathrm{Z})$ according to the following power law distribution $[1,2]$ :

$P(z)=\left(P_{t}-P_{b}\right) V_{t}+P_{b}$

where $P_{t}$ and $P_{b}$ are respectively the material properties at the top and bottom surfaces of the FGB, $V_{t}$ is the volume fraction of the top constituent of the beam defined as:

$V_{t}=\left(\frac{z}{h}+\frac{1}{2}\right)^{k} \quad(k \geq 0)$

In Eq. (2), $k$ is the power law index which dictates the material variation profile through the beam thickness. Figure 2 illustrates the variation of the volume fraction of the top constituent $\left(V_{t}\right)$ through the beam thickness in terms of $k$. There are three special cases. Clearly $k=1$ indicates a linear variation of properties between the top and bottom surfaces, $k=0$ represents a FGB made of full material of the top surface and infinite $k$ represents the beam made of full material of the bottom surface. 
Displacements $u_{1}, v_{1}$ and $w_{1}$ along the $\mathrm{X}, \mathrm{Y}$ and $\mathrm{Z}$ directions of a point on the cross-section are given by:

$u_{1}=0, \quad v_{1}(y, z, t)=v(y, t)-z \frac{\partial w(y, t)}{\partial y}+\varphi(z) \psi(y, t), \quad w_{1}(y, z, t)=w(y, t)$

where $v$ and $w$ are the corresponding displacements of a point on the neutral axis of the beam. In Eq. (3), $\varphi(z)$ characterises the distribution of the transverse shear stress through the beam thickness which can be described using different beam theories. In the current investigation, the Timoshenko beam theory (TBT) is applied which assumes constant shear stress and shear strain through the beam cross-section. Thus,

$$
\varphi(z)=z
$$

The transverse shear strain $\psi(z)$ at any point on the neutral axis of the beam is:

$\psi(y, t)=\frac{\partial w(y, t)}{\partial y}-\phi(y, t)$

where $\phi$ is the total bending rotation of the cross-sections at any point on the neutral axis of the beam and is taken as an unknown function. The displacement $v_{1}$ with the help of Eq. (5) becomes

$$
v_{1}(y, z, t)=v(y, t)-z \phi(y, t)
$$

The normal and shear strains [27] in the usual notation are:

$\varepsilon_{y y}=\frac{\partial v_{1}}{\partial y}=\frac{\partial v}{\partial y}-z \frac{\partial \phi}{\partial y}, \quad \gamma_{y z}=\frac{\partial v_{1}}{\partial z}+\frac{\partial w_{1}}{\partial y}=\frac{\partial w}{\partial y}-\phi$

Assuming that the material of the FGB obeys Hooke's law, the normal and shear stresses of the beam can be obtained as:

$\sigma_{y y}=E(z) \varepsilon_{y y}, \quad \tau_{y z}=G(z) \gamma_{y z}$ 
Using the above constitutive relationships, the potential energy $U$ and kinetic energy $T$ of the FGB are given by [23]

$$
\begin{aligned}
U & =\frac{1}{2} \int_{y y}\left(\sigma_{y y} \varepsilon_{y z}+\tau_{y z} \gamma_{y z}\right) d V \\
& =\frac{1}{2} \int_{0}^{L}\left\{A_{0} v^{\prime 2}-2 A_{1} v^{\prime} \phi^{\prime}+A_{2} \phi^{\prime 2}\right\} d y+\frac{1}{2} \int_{0}^{L} A_{3}\left\{w^{\prime 2}-2 w^{\prime} \phi+\phi^{2}\right\} d y \\
T & =\frac{1}{2} \int_{0}^{L}\left\{\int \rho(z)\left(\dot{v}_{1}^{2}+\dot{w}_{1}^{2}\right)\right\} d A d y=\frac{1}{2} \int_{0}^{L}\left\{I_{0}\left(\dot{v}^{2}+\dot{w}^{2}\right)-2 I_{1} \dot{v} \dot{\phi}+I_{2} \dot{\phi}^{2}\right\} d y
\end{aligned}
$$

where a prime and an over-dot represent differentiation with respect to space $y$ and time $t$ respectively. The parameters $I_{i}(i=0,1,2)$ and $A_{j}(j=0,1,2,3)$ are defined as:

$$
I_{i}=\int z^{i} \rho(z) d A, \quad A_{i}=\int z^{i} E(z) d A \quad(i=0,1,2), \quad A_{3}=\int G(z) d A
$$

Hamilton's principle states

$$
\delta \int_{t_{1}}^{t_{2}}(T-U) d t=0
$$

where $t_{1}$ and $t_{2}$ are the time interval in the dynamic trajectory, and $\delta$ is the usual variational operator.

The governing differential equations of motion and natural boundary conditions in free vibration are obtained by substituting the potential and kinetic energies into Eq. (12), using the $\delta$ operator, integrating by parts and then by collecting terms and noting that $\delta v, \delta w$ and $\delta \phi$ are completely arbitrary. The procedure to derive the governing differential equations and natural boundary conditions for beam structures has been processed through the application of symbolic computation [25]. The following governing differential equations of motion are eventually derived 


$$
\begin{aligned}
& -I_{0} \ddot{v}+A_{0} v^{\prime \prime}+I_{1} \ddot{\phi}-A_{1} \phi^{\prime \prime}=0 \\
& -I_{0} \ddot{w}+A_{3} w^{\prime \prime}-A_{3} \phi^{\prime}=0 \\
& I_{1} \ddot{v}-A_{1} v^{\prime \prime}+A_{3} w^{\prime}-I_{2} \ddot{\phi}+A_{2} \phi^{\prime \prime}-A_{3} \phi=0
\end{aligned}
$$

The natural boundary conditions are obtained in the process as a by-product of the Hamiltonian formulation. Therefore, the axial force $F$, shear force $S$ and bending moment $M$ are obtained as

$$
F=-A_{0} v^{\prime}+A_{1} \phi^{\prime}, \quad S=-A_{3} w^{\prime}+A_{3} \phi, \quad M=A_{1} v^{\prime}-A_{2} \phi^{\prime}
$$

Clearly the axial and bending motions are coupled due to the use of FGM in the Timoshenko beam formulation as evident from Eqs. (13) to (16).

Assuming harmonic oscillation so that

$$
v(y, t)=V(y) e^{i \omega t}, \quad w(y, t)=W(y) e^{i \omega t}, \quad \phi(y, t)=\Phi(y) e^{i \omega t}
$$

where $V(y), W(y)$ and $\Phi(y)$ are amplitudes of $v, w$ and $\phi$, and $\omega$ is the angular or circular frequency.

Introducing the differential operator $D=d / d \xi$ and the non-dimensional length $\xi$ as:

$$
\xi=y / L
$$

the differential equations of motion in Eqs. (13) - (15) can be written as:

$$
\begin{aligned}
& I_{0} \omega^{2} L^{2} V(\xi)+A_{0} D^{2} V(\xi)-I_{1} \omega^{2} L^{2} \Phi(\xi)-A_{1} D^{2} \Phi(\xi)=0 \\
& I_{0} \omega^{2} L^{2} W(\xi)+A_{3} D^{2} W(\xi)-A_{3} L D \Phi(\xi)=0 \\
& -I_{1} \omega^{2} L^{2} V(\xi)-A_{1} D^{2} V(\xi)+A_{3} L D W(\xi)+\left(I_{2} \omega^{2}-A_{3}\right) L^{2} \Phi(\xi)+A_{2} D^{2} \Phi(\xi)=0
\end{aligned}
$$


The above three equations can be combined into one sixth order ordinary differential equation, which satisfies each of $V(\xi), W(\xi)$ and $\Phi(\xi)$ so as to give

$\left(D^{6}+a D^{4}+b D^{2}+c\right) H=0$

where

$H=V(\xi)$ or $W(\xi)$ or $\Phi(\xi)$

and the frequency dependent co-efficients $a, b$ and $c$ are given by

$$
\begin{aligned}
& a=\frac{A_{0} A_{2} I_{0}-A_{1}^{2} I_{0}+A_{0} A_{3} I_{2}-2 A_{1} A_{3} I_{1}+A_{2} A_{3} I_{0}}{\left(A_{0} A_{2}-A_{1}^{2}\right) A_{3}} L^{2} \omega^{2} \\
& b=\frac{A_{2} I_{0}^{2} \omega^{2}-A_{3} I_{1}^{2} \omega^{2}-A_{0} A_{3} I_{0}-2 A_{1} I_{0} I_{1} \omega^{2}+A_{0} I_{0} I_{2} \omega^{2}+A_{3} I_{0} I_{2} \omega^{2}}{\left(A_{0} A_{2}-A_{1}^{2}\right) A_{3}} L^{4} \omega^{2} \\
& c=\frac{-I_{1}^{2} \omega^{2}+I_{0} I_{2} \omega^{2}-A_{3} I_{0}}{\left(A_{0} A_{2}-A_{1}^{2}\right) A_{3}} L^{6} \omega^{4} I_{0}
\end{aligned}
$$

The characteristic or auxiliary equation of the differential equation given by Eq. (22) can be reduced to a cubic equation which is amenable to analytical solution using standard procedure [28]. By taking the square root of the three roots of the cubic which could be real or complex, the six roots $r_{j}(j=1,2, \ldots, 6)$ of the auxiliary equation can be computed. Therefore, the solutions of the differential equations can be obtained as:

$$
V(\xi)=\sum_{j=1}^{6} P_{j} e^{r_{j} \xi}, \quad W(\xi)=\sum_{j=1}^{6} Q_{j} e^{r_{j} \xi}, \quad \Phi(\xi)=\sum_{j=1}^{6} R_{j} e^{r_{j} \xi}
$$

where $P_{j}, Q_{j}$ and $R_{j}(j=1,2, \ldots, 6)$ are three sets of six constants which are not all independent as they can be related to each other using Eqs. (19) - (21). The choice of relating two sets of the six constants in terms of the third one is arbitrary. 
Here, $R_{j}$ is chosen to be the base set of constants to be related with $P_{j}$ and $Q_{j}$. By substituting Eq. (25) into Eqs. (19) - (21), the following relationships are derived $P_{j}=\alpha_{j} R_{j}, \quad Q_{j}=\beta_{j} R_{j}$

where

$\alpha_{j}=\frac{I_{1} \omega^{2} L^{2}+A_{1} r_{j}^{2}}{I_{0} \omega^{2} L^{2}+A_{0} r_{j}^{2}}, \quad \beta_{j}=\frac{A_{3} L r_{j}}{I_{0} \omega^{2} L^{2}+A_{3} r_{j}^{2}}$

Similarly the amplitudes of the axial force $(F)$, shear force $(S)$ and bending moment $(M)$ are obtained in terms of the constant $R_{j}$ as follows:

$$
\begin{aligned}
& F=\frac{1}{L}\left(A_{1} \Phi^{\prime}-A_{0} V^{\prime}\right)=\frac{1}{L}\left(A_{1} \sum_{j=1}^{6} r_{j}-A_{0} \sum_{j=1}^{6} \alpha_{j} r_{j}\right) R_{j} e^{r_{j} \xi} \\
& S=\frac{A_{3}}{L}\left(-W^{\prime}+L \Phi\right)=\frac{A_{3}}{L}\left(L-\sum_{j=1}^{6} \beta_{j} r_{j}\right) R_{j} e^{r_{j} \xi} \\
& M=\frac{1}{L}\left(A_{1} V^{\prime}-A_{2} \Phi^{\prime}\right)=\frac{1}{L}\left(A_{1} \sum_{j=1}^{6} \alpha_{j} r_{j}-A_{2} \sum_{j=1}^{6} r_{j}\right) R_{j} e^{r_{j} \xi}
\end{aligned}
$$

Clearly $F, S$ and $M$ above are frequency-dependent as a consequence of Eq. (27).

The constants $R_{j}$ can now be written as a column matrix $\mathbf{R}$ to give:

$$
\mathbf{R}=\left[\begin{array}{llllll}
R_{1} & R_{2} & R_{3} & R_{4} & R_{5} & R_{6}
\end{array}\right]^{\mathrm{T}}
$$

where the upper suffix $\mathrm{T}$ denotes a transpose.

\subsection{Dynamic stiffness formulation}

The dynamic stiffness matrix of the FGB is derived by applying natural boundary conditions for displacements and forces at the ends of the beam. Figure 3 shows the sign convention used for axial force, shear force and bending moment when 
applying for the boundary conditions. The boundary conditions for the displacements and forces at the ends of the FGB shown in Fig. 4 are

At $y=0(\xi=0): V=V_{1}, W=W_{1}, \Phi=\Phi_{1}, F=F_{1}, S=S_{1}, M=M_{1}$

At $y=L(\xi=1): V=V_{2}, W=W_{2}, \Phi=\Phi_{2}, F=-F_{2}, S=-S_{2}, M=-M_{2}$

The displacement vector $\boldsymbol{\delta}$ and the force vector $\mathbf{P}$ can be expressed as:

$\boldsymbol{\delta}=\left[\begin{array}{llllll}V_{1} & W_{1} & \Theta_{1} & V_{2} & W_{2} & \Theta_{2}\end{array}\right]^{\mathrm{T}}, \quad \mathbf{P}=\left[\begin{array}{llllll}F_{1} & S_{1} & M_{1} & F_{2} & S_{2} & M_{2}\end{array}\right]^{\mathrm{T}}$

Substituting the boundary condition relationships in Eq. (32) into Eqs (25) and (26), the relationship between $\boldsymbol{\delta}$ and $\mathbf{R}$ can be derived as

$\boldsymbol{\delta}=\mathbf{B} \mathbf{R}$

where

$\mathbf{B}=\left[\begin{array}{llllll}\alpha_{1} & \alpha_{2} & \alpha_{3} & \alpha_{4} & \alpha_{5} & \alpha_{6} \\ \beta_{1} & \beta_{2} & \beta_{3} & \beta_{4} & \beta_{5} & \beta_{6} \\ 1 & 1 & 1 & 1 & 1 & 1 \\ \alpha_{1} e^{r_{1}} & \alpha_{2} e^{r_{2}} & \alpha_{3} e^{r_{3}} & \alpha_{4} e^{r_{4}} & \alpha_{5} e^{r_{5}} & \alpha_{6} e^{r_{6}} \\ \beta_{1} e^{r_{1}} & \beta_{2} e^{r_{2}} & \beta_{3} e^{r_{3}} & \beta_{4} e^{r_{4}} & \beta_{5} e^{r_{5}} & \beta_{6} e^{r_{6}} \\ e^{r_{1}} & e^{r_{2}} & e^{r_{3}} & e^{r_{4}} & e^{r_{5}} & e^{r_{6}}\end{array}\right]$

Similarly substituting the boundary conditions in Eq. (33) into Eqs. (28)-(30), the relationship between $\mathbf{P}$ and $\mathbf{R}$ can be derived as

$\mathbf{P}=\mathbf{A} \mathbf{R}$

where each element of the matrix $\mathbf{A}$ for $j=1,2, \ldots, 6$ is given by

$$
\left.\begin{array}{ll}
a_{1 j}=\frac{1}{L}\left(A_{1}-A_{0} \alpha_{j}\right) r_{j}, & a_{2 j}=\frac{A_{3}}{L}\left(L-r_{j} \beta_{j}\right), \\
a_{3 j}=\frac{1}{L}\left(A_{1} \alpha_{j}-A_{2}\right) r_{j}, & a_{4 j}=-\frac{1}{L}\left(A_{1}-A_{0} \alpha_{j}\right) r_{j} e^{r_{j}}, \\
a_{5 j}=-\frac{A_{3}}{L}\left(L-r_{j} \beta_{j}\right) e^{r_{j}}, & a_{6 j}=-\frac{1}{L}\left(A_{1} \alpha_{j}-A_{2}\right) r_{j} e^{r_{j}}
\end{array}\right\}
$$


To derive the dynamic stiffness matrix $\mathbf{K}, \mathbf{P}$ and $\boldsymbol{\delta}$ are to be related by eliminating the constant vector $\mathbf{R}$ in Eqs. (35) and (37) to give

$$
\mathbf{P}=\mathbf{K} \boldsymbol{\delta}
$$

where

$$
\mathbf{K}=\mathbf{A} \mathbf{B}^{-1}
$$

is the required $6 \times 6$ frequency dependent dynamic stiffness matrix.

The dynamic stiffness matrix $\mathbf{K}$ can now be used to compute natural frequencies and mode shapes of either an individual FGB or an assembly of FGBs with various boundary conditions. A reliable and accurate method of computing the natural frequencies using the DSM is to apply the well-established algorithm of Wittrick and Williams [26] generally known as the W-W algorithm in the literature which is ideally suited to solve transcendental (nonlinear) eigenvalue problems as in the present case. The algorithm uses the Sturm sequence property of the dynamic stiffness matrix and has featured in literally hundreds of papers. It ensures that no natural frequencies of the structure being analysed are missed. A brief explanation of the working principle of the $\mathrm{W}-\mathrm{W}$ algorithm is given below.

\section{Application of the Wittrick-Williams (W-W) algorithm}

The dynamic stiffness matrix of Eq. (40) can now be used to compute the natural frequencies and mode shapes of FGBs with various end conditions. A non-FGB can also be analysed for its free vibration characteristics by idealising it as an assemblage of many uniform FGBs. Before applying the W-W algorithm the dynamic stiffness matrices of all individual elements in the structure are to be 
assembled to form the overall dynamic stiffness matrix $\boldsymbol{K}_{f}$ of the final (complete) structure, which may, of course, consist of a single element. The algorithm (unlike its proof) is very simple to use. The procedure is briefly summarised as follows.

Suppose that $\omega$ denotes the circular (or angular) frequency of a vibrating structure. Then according to the $\mathrm{W}-\mathrm{W}$ algorithm [26], $j$, the number of natural frequencies passed, as $\omega$ is increased from zero to $\omega^{*}$, is given by

$$
j=j_{0}+\mathrm{s}\left\{\boldsymbol{K}_{f}\right\}
$$

where $\boldsymbol{K}_{f}$, the overall dynamic stiffness matrix of the final structure whose elements all depend on $\omega$, is evaluated at $\omega=\omega^{*} ; \mathrm{s}\left\{\boldsymbol{K}_{f}\right\}$ is the number of negative elements on the leading diagonal of $\boldsymbol{K}_{f}^{\Delta}, \boldsymbol{K}_{f}^{\Delta}$ being the upper triangular matrix obtained by applying the usual form of Gauss elimination to $\boldsymbol{K}_{f}$, and $j_{0}$ is the number of natural frequencies of the structure still lying between $\omega=0$ and $\omega=\omega^{*}$ when the displacement components to which $\boldsymbol{K}_{f}$ corresponds are all zeros. (Note that the structure can still have natural frequencies when all its nodes are clamped, because exact member equations allow each individual member to displace between nodes with an infinite number of degrees of freedom, and hence infinite number of natural frequencies between nodes.) Thus

$$
j_{0}=\sum j_{m}
$$


where $j_{m}$ is the number of natural frequencies between $\omega=0$ and $\omega=\omega^{*}$ for a component member with its ends fully clamped, while the summation extends over all members of the structure. For the element dynamic stiffness matrix developed in this paper, the clamped-clamped natural frequencies of an individual member are given by $\Delta=0$, where $\Delta$ is the determinant of the matrix $\mathbf{B}$ of Eq. (36). Thus, with the knowledge of Eqs. (41) and (42), it is possible to ascertain how many natural frequencies of a structure lie below an arbitrarily chosen trial frequency. This simple feature of the algorithm (coupled with the fact that successive trial frequencies can be chosen by the user to bracket a natural frequency) can be used to converge upon any required natural frequency to any desired (or specified) accuracy.

\section{Numerical results and discussions}

The theory developed in this paper is sufficiently general and thus can be used for any constituent materials comprising the FGB. Natural frequencies and mode shapes of a number of different FGBs are computed using a range of the material variation in the beam thickness direction. A substantial amount of validation exercises was needed to verify the accuracy of the method. It was thus necessary to compare results with those available in the literature. A parametric study is then carried out by varying the power law index $k$, the length to thickness ratio $L / h$, and the boundary conditions of the beam. Boundary conditions that are investigated include simply supported-simply supported (SS), clamped-clamped (CC), clamped-simply supported (CS) and clamped-free (CF). 
In order to make the results universal, the non-dimensional natural frequency parameter $\lambda_{i}$ is defined as follows:

$\lambda_{i}=\frac{\omega_{i} L^{2}}{h} \sqrt{\rho_{b} / E_{b}}$

where $\omega_{i}$ is the $i^{\text {th }}$ angular or circular natural frequency, $\rho_{b}$ and $E_{b}$ are the density and Young's modulus of the bottom surface material of the FGB.

First, the degenerated case when the FGB is made of pure Aluminium (Al) is investigated using the power law index $k=\infty$. For computational purposes, $k=10^{6}$ was used to represent the case for the unattainable $k=\infty$. This value of $k$ was sufficiently large to give the prescribed accuracy and yet avoided any numerical instability. Table 1 shows the non-dimensional fundamental natural frequency of the beam for simply-supported boundary conditions for three values of $L / h$ alongside the ones reported in Ref [17]. Two sets of results using the Timoshenko beam theory (TBT) and the classical beam theory (CBT) are also compared. The agreement between the sets of results from the present theory and those of Ref [17] is generally very good. The discrepancy between the TBT and CBT results is quite small, particular at higher values of $L / h$. The fundamental natural frequency increases with the increase in the ratio $L / h$ as expected.

Next a FGB constructed from $\mathrm{Al}$ and alumina $\left(\mathrm{Al}_{2} \mathrm{O}_{3}\right)$ in the bottom and top surfaces respectively, is used. The cross-sectional dimensions and material properties of the beam are taken from Ref [19] which are: 
$b=0.1 \mathrm{~m}, h=0.125 \mathrm{~m}$

$\mathrm{Al}_{2} \mathrm{O}_{3}$ (top surface): $\quad E_{t}=380 \mathrm{GPa}, \rho_{t}=3800 \mathrm{~kg} / \mathrm{m}^{3}, \quad v_{t}=0.23$

$\mathrm{Al}$ (bottom surface): $\quad E_{b}=70 \mathrm{GPa}, \quad \rho_{t}=2700 \mathrm{~kg} / \mathrm{m}^{3}, \quad v_{b}=0.23$

Generally the Poisson's ratio of material does not vary too much and it is kept constant in the analysis although the theory developed can accommodate for its variation. Shear correction or shape factor required by the TBT in the present analysis was set to $\kappa=5 / 6$ for the rectangular cross-section so as to be consistent with the same value used in the literature $[10,16]$. As in the case of previous investigations $[10,16]$, this factor has been kept constant in this analysis, but it can be absorbed within the shear modulus $(G)$ or the Young's modulus $(E)$ which follows appropriate property variations. The following non-dimensional frequency parameter defined in Ref [19] is used so as to make the results directly comparable:

$$
\lambda_{i}=\frac{\omega_{i} L^{2}}{h} \sqrt{\frac{I_{0}}{A_{0}}}
$$

where $I_{0}$ and $A_{0}$ have already been defined in Eq. (11).

Table 2 shows the non-dimensional fundamental natural frequency of an FGB with different boundary conditions for $L / h=10$ and $k=0.3$ alongside the results of Refs [17] and [19]. Excellent agreement is achieved between the sets of results as can be seen in the table. 
Then next set of results is obtained for a FGB made of steel and $\mathrm{Al}_{2} \mathrm{O}_{3}$ representing the bottom and top surfaces, respectively. A different value of the power law index parameter, i.e. $k=1$ as opposed to $k=0.3$ of the above example, is used this time to make the results directly comparable with Ref [5]. The cross-sectional dimensions, density, Young's Modulus and Poisson's ratio of the beam are taken from Ref [5] as:

$b=0.1 \mathrm{~m}, h=0.1 \mathrm{~m}$

Steel (bottom surface): $E_{b}=210 \mathrm{GPa}, \rho_{b}=7800 \mathrm{~kg} / \mathrm{m}^{3}, v_{b}=0.31$,

$\mathrm{Al}_{2} \mathrm{O}_{3}$ (top surface): $E_{t}=390 \mathrm{GPa}, \rho_{t}=3960 \mathrm{~kg} / \mathrm{m}^{3}, v_{t}=0.25$.

To be consistent with Ref [5], a different non-dimensional frequency parameter is now defined as:

$\lambda_{i}=100 \omega_{i} h \sqrt{E_{b} / \rho_{b}}$

Table 3 shows the non-dimensional fundamental natural frequencies of the FGB for three values of the ratio $L / h$ for the case $k=1$ corresponding to the SS boundary conditions alongside the results reported in Ref [5]. Excellent agreement is achieved as can be seen in the table.

The above validation exercises confirmed the correctness and predictable accuracy of the DSM developed in this paper. Next, a parametric investigation by varying significant FGB parameters and boundary conditions is carried out. The crosssectional dimensions, the material constitution of the bottom and top surfaces of the FGB are kept the same as used in Ref [5]. The natural frequencies of the FGB are computed for a range of values of the ratio $L / h$ and the power law index $k$ for the 
four classical boundary conditions. In particular, the effects of the $L / h$ ratio and the $k$ parameter on the natural frequencies and mode shapes are given precedence when obtaining the results. Numerical results are non-dimensionalised using Eq. (43).

Tables 4 to 7 show the first five non-dimensional natural frequencies of the FGB for the SS, CC, CS and CF boundary conditions, respectively. It can be seen that all the natural frequency decreases with the increase in $k$ for all four classical boundary conditions. This is to be expected because the material properties tend towards those of steel as $k$ increases for which $E / \rho$ is much smaller than alumina. Naturally, the highest natural frequencies are obtained for the case when the beam is made of almost ceramic $\mathrm{Al}_{2} \mathrm{O}_{3}$, i.e. $k$ tends towards zero whereas the lowest ones are obtained for the case when the FGB is almost metal (Steel), i.e. $k$ tends towards infinity.

The natural frequencies increase when the ratio $L / h$ increases for a fixed value of $k$, as expected. It can be seen that there is no significant change on the fundamental natural frequency when the ratio $L / h$ assumes higher values, for which the CBT is probably adequate, see $L / h=30$ and 100 .

In order to establish trends, the effect of the $L / h$ ratio on the fundamental natural frequency is shown graphically in Fig. 5 for a set of $k$ values for cantilever boundary condition of the FGB. It can be seen that the fundamental natural frequency changes significantly when $L / h \leq 10$ as expected. 
To illustrate the effect of $k$ on natural frequencies, Fig. 6 show the first three natural frequencies of a cantilever FGB for three values of $L / h=5,10,100$. The fundamental natural frequency is reduced as the parameter $k$ increases from zero when the FGB is made from pure alumina and approaches towards large values corresponding to the top surface steel properties. The same pattern was observed for all three values of the $L / h$ ratio. The second and third natural frequencies show similar trend but with significantly pronounced effect of $L / h$ due to the variation of $k$.

In order to compare results obtained using the present TBT with the CBT, Fig. 7 shows the first two non-dimensional natural frequencies of a cantilever FGB for $L / h$ $=10$. Clearly the results using the TBT give lower values than the ones using the CBT as expected, but for the fundamental natural frequency the results are virtually the same. The maximum discrepancy in results using the CBT and TBT is around 4\% occurring in the second natural frequency.

The final set of results was obtained to demonstrate the mode shapes of the FGB. Figs. 8(a) and 8(b) show the first five normalised mode shapes of the cantilever FGB when $k=1$ for $L / h=10$ and 20 respectively. The investigation has shown that there was no significant effect on the mode shapes with respect to the variation of the power law index $k$. This may be due to the fact that material properties vary through the thickness direction only as opposed to the axial direction. Referring to Fig. 8, the first two modes are dominated by bending displacements for both $L / h=10$ and 20. The third mode is essentially an axial mode when $L / h=10$ whereas it becomes 
a bending mode for $L / h=20$. By contrast, an axial mode is prevalent for the fourth mode when $L / h=20$ whereas it is essentially a bending mode when $L / h=10$. The fifth mode becomes a bending mode again for both values of $L / h=10$ and 20 . For the two values of the $L / h$ ratio used in the figures, modal interchanges between the third and fourth modes occur as a result of using FGM in conjunction with the Timoshenko beam theory. The occurrence of such modal interchanges (or modal flip-over) is very interesting and it can be useful in solving frequency attenuation problems.

\section{Conclusions}

Starting from the derivation of the governing differential equations of motion in free vibration, the dynamic stiffness matrix of a functionally graded Timoshenko beam has been developed and applied with particular reference to the Wittrick-Williams algorithm to investigate its free vibration characteristics. Natural frequencies and mode shapes of some illustrative examples are illustrated and compared with published ones wherever possible. The investigation has revealed that by choosing the material distribution law and the length to thickness ratio in an appropriate way, it is possible to alter the natural frequencies and mode shapes of a FGB in a significant way. This is particularly useful in solving frequency attenuation problems. The proposed method is computationally efficient and numerically accurate. The method gives exact results and can be used as an aid to validate finite element and other approximate methods. 


\section{References}

[1] Alshorbagy AE, Eltaher MA, Mahmoud FF. Free vibration characteristics of a functionally graded beam by finite element method. Appl Math Model 2011;35:412-425.

[2] Aydogdu M, Taskin V. Free vibration analysis of functionally graded beams with simply supported edges. Mater Design 2007;28:1651-1656.

[3] Bîrsan M, Altenbach H, Sadowski T, Eremeyev VA, Pietras D. Deformation analysis of functionally graded beams by the direct approach. Compos Part BEng 2012;43:1315-1328.

[4] Chakraborty A, Gopalakrishnan S, Reddy JN. A new beam finite element for the analysis of functionally graded materials. Int J Mech Sci 2003;45:519-539.

[5] Giunta G, Crisafulli D, Belouettar S, Carrera E. Hierarchical theories for the free vibration analysis of functionally graded beams. Compos Struct 2011;94:68-74.

[6] Huang Y, Li XF. A new approach for free vibration analysis of axially functionally graded beams with non-uniform cross-section. J Sound Vib 2010;329:2291-2303.

[7] Huang Y, Yang LE, Luo QZ. Free vibration of axially functionally graded Timoshenko beams with non-uniform cross-section. Compos Part B-Eng 2013;45:1493-1498.

[8] Kapuria S, Bhattacharyya M, Kumar AN. Bending and free vibration response of layered functionally graded beams: A theoretical model and its experimental validation, Compos Struct 2008;82:390-402. 
[9] Lai SK, Harrington J, Xiang Y, Chow KW. Accurate analytical perturbation approach for large amplitude vibration of functionally graded beams. Int $\mathbf{J}$ Nonlinear Mech 2012; 47:473-480.

[10] Li XF. A unified approach for analyzing static and dynamic behaviors of functionally graded Timoshenko and Euler-Bernoulli beams. J Sound Vib $2008 ; 318: 1210-1229$.

[11] Librescu L, Oh SY, Song O. Thin-walled beams made of functionally graded materials and operating in a high temperature environment: vibration and stability. J Therm Stress 2005;28:649-712.

[12] Loja MAR, Barbosa JI, Mota Soares CM. A study on the modelling of sandwich functionally graded particulate composite. Compos Struct 2012;94:2209-2217.

[13] Lu CF, Chen WQ. Free vibration of orthotropic functionally graded beams with various end conditions. Struct Eng Mech 2005;20:465-476.

[14] Oh SY, Librescu L, Song O. Vibration of turbomachinery rotating blades made-up of functionally graded materials and operating in a high temperature field. Acta Mech 2003;166:69-87.

[15] Sankar BV. An elasticity solution for functionally graded beams. Compos Sci Tech 2001;61:689-696.

[16] Simsek M. Fundamental frequency analysis of functionally graded beams by using different higher-order beam theories. Nucl Eng Des 2010;240:697-705.

[17] Sina SA, Navazi HM, Haddadpour H. An analytical method for free vibration analysis of functionally graded beams. Mater Design 2009;30:741-747. 
[18] Thai HT, Vo TP. Bending and free vibration of functionally graded beams using various higher-order shear deformation beam theories. Int J Mech Sci 2012;62:57-66.

[19] Wattanasakulpong N, Prusty BG, Kelly DW, Hoffman M. Free vibration analysis of layered functionally graded beams with experimental validation. Mater Design 2012;36:182-190.

[20] Xiang HJ, Yang J. Free and forced vibration of a laminated FGM Timoshenko beam of variable thickness under heat conduction. Compos Part B-Eng 2008;39:292-303.

[21] Zhong Z, Yu T, Analytical solution of a cantilever functionally graded beam. Compos Sci Tech 2007;67:481-488.

[22] Zhu H, Sankar BV. A combined Fourier series-Galerkin method for the analysis of functionally graded beams. J Appl Mech Trans ASME 2004;71:421-424.

[23] $\mathrm{Su} \mathrm{H}$, Banerjee JR. Free vibration of a functionally graded Timoshenko beam using the dynamic stiffness method. Proceedings of the 11th Int Conf on Comput Struct Tech, Civil-Comp Press, Stirlingshire, UK, 2012, Paper No 102, DOI: $10.4203 /$ ccp.99.102.

[24] Banerjee JR. Free vibration of sandwich beams using the dynamic stiffness method. Comput Struct 2003;81:1915-1922.

[25] Banerjee JR, Sobey AJ, Su H, Fitch JP, Use of computer algebra in Hamiltonian calculations. Adv Eng Softw 2008;39:521-525. 
[26] Wittrick WH, Williams FW. A general algorithm for computing natural frequencies of elastic structures. Q J Mech Appl Math 1971;24:263-284.

[27] Timoshenko SP, Goodier JN. Theory of elasticity, McGraw-Hill; 1970.

[28] Pipes LA, Harvill LR. Applied mathematics for engineers and physicists, McGraw-Hill; 1970. 
Table 1. Non-dimensional fundamental natural frequency of a pure Al beam with SS boundary conditions

\begin{tabular}{|c|c|c|c|c|}
\hline \multirow{3}{*}{$L / h$} & \multicolumn{4}{|c|}{$\begin{array}{l}\text { Non-dimensional fundamental natural frequency } \\
\qquad \lambda_{1}=\frac{\omega_{1} L^{2}}{h} \sqrt{\rho_{b} / E_{b}}\end{array}$} \\
\hline & \multicolumn{2}{|c|}{ Current theory } & \multicolumn{2}{|c|}{$\operatorname{Ref}[17]$} \\
\hline & TBT & CBT & TBT & CBT \\
\hline 10 & 2.8023 & 2.8375 & 2.797 & 2.849 \\
\hline 30 & 2.8439 & 2.8478 & 2.843 & 2.849 \\
\hline 100 & 2.8496 & 2.8496 & 2.848 & 2.849 \\
\hline
\end{tabular}


Table 2. Non-dimensional fundamental natural frequency $\lambda_{1}$ of a FGB for $L / h=10$ and $k=0.3$ with various boundary conditions

\begin{tabular}{|c|c|c|c|}
\hline \multirow{2}{*}{ BCs } & \multicolumn{3}{|c|}{ Non-dimensional fundamental natural frequency } \\
& & $\lambda_{1}=\frac{\omega_{1} L^{2}}{h} \sqrt{\frac{I_{0}}{A_{0}}}$ \\
\hline & Current theory & $\operatorname{Ref}[17]$ & $\operatorname{Ref}[19]$ \\
\hline $\mathrm{SS}$ & 2.7450 & 2.774 & 2.803 \\
\hline $\mathrm{CC}$ & 5.9544 & 6.013 & 6.078 \\
\hline $\mathrm{CF}$ & 0.9858 & 0.996 & 1.008 \\
\hline $\mathrm{CS}$ & 4.2030 & - & 4.291 \\
\hline
\end{tabular}


Table 3. The non-dimensional fundamental natural frequency of an FGB with SS boundary conditions when $k=1$

\begin{tabular}{|c|c|c|c|c|}
\hline \multirow{3}{*}{$L / h$} & \multicolumn{4}{|c|}{$\begin{array}{c}\text { Non-dimensional fundamental natural frequency } \\
\left(\lambda_{1}=100 \omega_{1} h \sqrt{E_{b} / \rho_{b}}\right)\end{array}$} \\
\cline { 2 - 5 } & \multicolumn{4}{|c|}{ Ref [5] } \\
\cline { 2 - 5 } & CBT & TBT & CBT & TBT \\
\hline 100 & 0.039218 & 0.039213 & 0.039219 & 0.039215 \\
\hline 10 & 3.9059 & 3.8586 & 3.9060 & 3.8663 \\
\hline 5 & 15.436 & 14.756 & 15.436 & 14.861 \\
\hline
\end{tabular}


Table 4. The first five non-dimensional natural frequencies of an FGB with SS boundary conditions for a range of values of $L / h$ and $k$

\begin{tabular}{|c|c|c|c|c|c|c|c|c|}
\hline \multirow[t]{2}{*}{$L / h$} & \multirow{2}{*}{$\begin{array}{c}\text { Freq } \\
\text { No. } \\
\\
{ }_{i}\end{array}$} & \multicolumn{7}{|c|}{$\begin{array}{l}\text { Non-dimensional natural frequencies } \\
\qquad\left(\lambda_{i}=\frac{\omega_{i} L^{2}}{h} \sqrt{\rho_{b} / E_{b}}\right)\end{array}$} \\
\hline & & $k=0.1$ & $k=0.2$ & $k=0.5$ & $k=1$ & $k=2$ & $k=5$ & $k=10$ \\
\hline \multirow{5}{*}{5} & 1 & 4.7840 & 4.5296 & 4.0590 & 3.6890 & 3.3906 & 3.1088 & 2.9513 \\
\hline & 2 & 16.652 & 15.770 & 14.128 & 12.818 & 11.740 & 10.721 & 10.176 \\
\hline & 3 & 28.189 & 26.780 & 24.022 & 21.621 & 19.479 & 17.526 & 16.686 \\
\hline & 4 & 31.924 & 30.239 & 27.085 & 24.531 & 22.387 & 20.366 & 19.331 \\
\hline & 5 & 48.579 & 46.026 & 41.215 & 37.269 & 33.912 & 30.757 & 29.194 \\
\hline \multirow{5}{*}{10} & 1 & 5.0010 & 4.7348 & 4.2432 & 3.8586 & 3.5510 & 3.2608 & 3.0959 \\
\hline & 2 & 19.136 & 18.118 & 16.235 & 14.755 & 13.561 & 12.434 & 11.805 \\
\hline & 3 & 40.385 & 38.240 & 34.261 & 31.110 & 28.544 & 26.122 & 24.799 \\
\hline & 4 & 56.379 & 53.561 & 48.044 & 43.242 & 38.958 & 35.052 & 33.371 \\
\hline & 5 & 66.608 & 63.075 & 56.502 & 51.256 & 46.941 & 42.873 & 40.700 \\
\hline \multirow{5}{*}{20} & 1 & 5.0613 & 4.7918 & 4.2943 & 3.9058 & 3.5957 & 3.3032 & 3.1363 \\
\hline & 2 & 20.004 & 18.939 & 16.972 & 15.433 & 14.202 & 13.042 & 12.383 \\
\hline & 3 & 44.156 & 41.805 & 37.460 & 34.052 & 31.319 & 28.743 & 27.291 \\
\hline & 4 & 76.542 & 72.468 & 64.928 & 58.997 & 54.220 & 49.725 & 47.214 \\
\hline & 5 & 112.76 & 107.12 & 96.090 & 86.486 & 77.917 & 70.104 & 66.743 \\
\hline \multirow{5}{*}{30} & 1 & 5.0727 & 4.8026 & 4.3041 & 3.9147 & 3.6042 & 3.3113 & 3.1440 \\
\hline & 2 & 20.181 & 19.107 & 17.123 & 15.572 & 14.334 & 13.167 & 12.502 \\
\hline & 3 & 45.009 & 42.611 & 38.184 & 34.719 & 31.950 & 29.342 & 27.861 \\
\hline & 4 & 79.058 & 74.847 & 67.063 & 60.962 & 56.077 & 51.484 & 48.888 \\
\hline & 5 & 121.70 & 115.22 & 103.22 & 93.801 & 86.244 & 79.149 & 75.163 \\
\hline \multirow{5}{*}{100} & 1 & 5.0811 & 4.8105 & 4.3112 & 3.9213 & 3.6104 & 3.3173 & 3.1496 \\
\hline & 2 & 20.314 & 19.232 & 17.236 & 15.676 & 14.433 & 13.261 & 12.591 \\
\hline & 3 & 45.670 & 43.237 & 38.745 & 35.236 & 32.440 & 29.808 & 28.304 \\
\hline & 4 & 81.097 & 76.775 & 68.793 & 62.556 & 57.587 & 52.918 & 50.253 \\
\hline & 5 & 126.53 & 119.78 & 107.32 & 97.572 & 89.812 & 82.537 & 78.388 \\
\hline
\end{tabular}


Table 5. The first five non-dimensional natural frequencies of an FGB with CC boundary conditions for a range of values of $L / h$ and $k$

\begin{tabular}{|c|c|c|c|c|c|c|c|c|}
\hline \multirow[t]{2}{*}{$L / h$} & \multirow{2}{*}{$\begin{array}{c}\text { Freq } \\
\text { No. } \\
\\
{ }_{i}\end{array}$} & \multicolumn{7}{|c|}{$\begin{array}{l}\text { Non-dimensional natural frequencies } \\
\qquad\left(\lambda_{i}=\frac{\omega_{i} L^{2}}{h} \sqrt{\rho_{b} / E_{b}}\right)\end{array}$} \\
\hline & & $k=0.1$ & $k=0.2$ & $k=0.5$ & $k=1$ & $k=2$ & $k=5$ & $k=10$ \\
\hline \multirow{5}{*}{5} & 1 & 9.3380 & 8.8467 & 7.9241 & 7.1772 & 6.5543 & 5.9699 & 5.6680 \\
\hline & 2 & 21.455 & 20.331 & 18.206 & 16.459 & 14.974 & 13.585 & 12.896 \\
\hline & 3 & 28.189 & 26.780 & 24.022 & 21.621 & 19.479 & 17.526 & 16.686 \\
\hline & 4 & 35.825 & 33.952 & 30.399 & 27.456 & 24.932 & 22.573 & 21.428 \\
\hline & 5 & 51.248 & 48.571 & 43.484 & 39.251 & 35.603 & 32.193 & 30.559 \\
\hline \multirow{5}{*}{10} & 1 & 10.827 & 10.253 & 9.1864 & 8.3437 & 7.6610 & 7.0184 & 6.6638 \\
\hline & 2 & 27.809 & 26.337 & 23.594 & 21.404 & 19.608 & 17.919 & 17.014 \\
\hline & 3 & 50.364 & 47.704 & 42.727 & 38.721 & 35.398 & 32.279 & 30.647 \\
\hline & 4 & 56.379 & 53.561 & 48.044 & 43.242 & 38.958 & 35.052 & 33.371 \\
\hline & 5 & 76.611 & 72.572 & 64.989 & 58.840 & 53.695 & 48.873 & 46.401 \\
\hline \multirow{5}{*}{20} & 1 & 11.334 & 10.731 & 9.6159 & 8.7425 & 8.0431 & 7.3844 & 7.0116 \\
\hline & 2 & 30.602 & 28.974 & 25.961 & 23.593 & 21.688 & 19.896 & 18.892 \\
\hline & 3 & 58.430 & 55.324 & 49.565 & 45.019 & 41.347 & 37.898 & 35.987 \\
\hline & 4 & 93.607 & 88.635 & 79.395 & 72.072 & 66.128 & 60.554 & 57.504 \\
\hline & 5 & 112.76 & 107.12 & 96.090 & 86.486 & 77.917 & 70.104 & 66.743 \\
\hline \multirow{5}{*}{30} & 1 & 11.436 & 10.827 & 9.7027 & 8.8232 & 8.1207 & 7.4591 & 7.0825 \\
\hline & 2 & 31.225 & 29.563 & 26.490 & 24.083 & 22.157 & 20.345 & 19.319 \\
\hline & 3 & 60.453 & 57.235 & 51.280 & 46.605 & 42.857 & 39.337 & 37.355 \\
\hline & 4 & 98.402 & 93.164 & 83.458 & 75.822 & 69.685 & 63.933 & 60.718 \\
\hline & 5 & 144.38 & 136.69 & 122.43 & 111.18 & 102.12 & 93.646 & 88.946 \\
\hline \multirow{5}{*}{100} & 1 & 11.513 & 10.899 & 9.7675 & 8.8835 & 8.1788 & 7.5149 & 7.1355 \\
\hline & 2 & 31.707 & 30.017 & 26.898 & 24.461 & 22.519 & 20.692 & 19.649 \\
\hline & 3 & 62.084 & 58.774 & 52.662 & 47.884 & 44.078 & 40.505 & 38.467 \\
\hline & 4 & 102.47 & 97.007 & 86.907 & 79.010 & 72.722 & 66.832 & 63.477 \\
\hline & 5 & 152.79 & 144.64 & 129.56 & 117.77 & 108.38 & 99.612 & 94.623 \\
\hline
\end{tabular}


Table 6. The first five non-dimensional natural frequencies of an FGB with CS boundary conditions for a range of values of $L / h$ and $k$

\begin{tabular}{|c|c|c|c|c|c|c|c|c|}
\hline \multirow[t]{2}{*}{$L / h$} & \multirow{2}{*}{$\begin{array}{c}\text { Freq } \\
\text { No. } \\
\\
{ }_{i}\end{array}$} & \multicolumn{7}{|c|}{$\begin{array}{l}\text { Non-dimensional natural frequencies } \\
\qquad\left(\lambda_{i}=\frac{\omega_{i} L^{2}}{h} \sqrt{\rho_{b} / E_{b}}\right)\end{array}$} \\
\hline & & $k=0.1$ & $k=0.2$ & $k=0.5$ & $k=1$ & $k=2$ & $k=5$ & $k=10$ \\
\hline \multirow{5}{*}{5} & 1 & 6.9523 & 6.5845 & 5.8992 & 5.3522 & 4.9032 & 4.4805 & 4.2537 \\
\hline & 2 & 19.153 & 18.144 & 16.252 & 14.717 & 13.431 & 12.222 & 11.602 \\
\hline & 3 & 28.189 & 26.780 & 24.022 & 21.621 & 19.479 & 17.526 & 16.686 \\
\hline & 4 & 33.951 & 32.169 & 28.807 & 26.051 & 23.711 & 21.514 & 20.422 \\
\hline & 5 & 49.968 & 47.351 & 42.396 & 38.300 & 34.791 & 31.503 & 29.903 \\
\hline \multirow{5}{*}{10} & 1 & 7.6505 & 7.2438 & 6.4912 & 5.8996 & 5.4236 & 4.9750 & 4.7235 \\
\hline & 2 & 23.402 & 22.161 & 19.855 & 18.029 & 16.544 & 15.145 & 14.379 \\
\hline & 3 & 45.404 & 43.000 & 38.519 & 34.942 & 32.000 & 29.232 & 27.753 \\
\hline & 4 & 56.379 & 53.561 & 48.044 & 43.242 & 38.958 & 35.052 & 33.371 \\
\hline & 5 & 71.694 & 67.903 & 60.818 & 55.116 & 50.383 & 45.934 & 43.609 \\
\hline \multirow{5}{*}{20} & 1 & 7.8636 & 7.4449 & 6.6719 & 6.0672 & 5.5838 & 5.1283 & 4.8692 \\
\hline & 2 & 25.073 & 23.739 & 21.272 & 19.337 & 17.787 & 16.326 & 15.502 \\
\hline & 3 & 51.128 & 48.408 & 43.373 & 39.412 & 36.223 & 33.224 & 31.547 \\
\hline & 4 & 84.981 & 80.462 & 72.083 & 65.467 & 60.118 & 55.092 & 52.314 \\
\hline & 5 & 112.76 & 107.12 & 96.090 & 86.486 & 77.917 & 70.104 & 66.743 \\
\hline \multirow{5}{*}{30} & 1 & 7.9052 & 7.4842 & 6.7072 & 6.0999 & 5.6152 & 5.1584 & 4.8978 \\
\hline & 2 & 25.429 & 24.075 & 21.574 & 19.617 & 18.053 & 16.581 & 15.744 \\
\hline & 3 & 52.490 & 49.695 & 44.528 & 40.479 & 37.237 & 34.189 & 32.465 \\
\hline & 4 & 88.535 & 83.820 & 75.096 & 68.245 & 62.750 & 57.591 & 54.691 \\
\hline & 5 & 132.89 & 125.81 & 112.70 & 102.38 & 94.088 & 86.314 & 81.974 \\
\hline \multirow{5}{*}{100} & 1 & 7.9359 & 7.5133 & 6.7332 & 6.1241 & 5.6384 & 5.1807 & 4.9190 \\
\hline & 2 & 25.700 & 24.331 & 21.804 & 19.830 & 18.256 & 16.775 & 15.928 \\
\hline & 3 & 53.567 & 50.712 & 45.441 & 41.322 & 38.041 & 34.956 & 33.194 \\
\hline & 4 & 91.480 & 86.603 & 77.593 & 70.550 & 64.941 & 59.679 & 56.677 \\
\hline & 5 & 139.36 & 131.93 & 118.19 & 107.44 & 98.889 & 90.883 & 86.323 \\
\hline
\end{tabular}


Table 7. The first five non-dimensional natural frequencies of an FGB with CF boundary conditions for a range of values of $L / h$ and $k$

\begin{tabular}{|c|c|c|c|c|c|c|c|c|}
\hline \multirow[t]{2}{*}{$L / h$} & $\begin{array}{l}\text { Freq } \\
\text { No. }\end{array}$ & \multicolumn{7}{|c|}{$\begin{array}{l}\text { Non-dimensional natural frequencies } \\
\qquad\left(\lambda_{i}=\frac{\omega_{i} L^{2}}{h} \sqrt{\rho_{b} / E_{b}}\right)\end{array}$} \\
\hline & $i$ & $k=0.1$ & $k=0.2$ & $k=0.5$ & $k=1$ & $k=2$ & $k=5$ & $k=10$ \\
\hline \multirow{5}{*}{5} & 1 & 1.7574 & 1.6638 & 1.4911 & 1.3557 & 1.2471 & 1.1446 & 1.0867 \\
\hline & 2 & 9.5011 & 8.9969 & 8.0609 & 7.3164 & 6.7053 & 6.1274 & 5.8159 \\
\hline & 3 & 14.095 & 13.390 & 12.012 & 10.811 & 9.7403 & 8.7633 & 8.3430 \\
\hline & 4 & 22.682 & 21.482 & 19.243 & 17.441 & 15.937 & 14.516 & 13.776 \\
\hline & 5 & 37.747 & 35.754 & 32.022 & 28.989 & 26.428 & 24.009 & 22.783 \\
\hline \multirow{5}{*}{10} & 1 & 1.7966 & 1.7010 & 1.5244 & 1.3864 & 1.2762 & 1.1722 & 1.1130 \\
\hline & 2 & 10.782 & 10.208 & 9.1477 & 8.3146 & 7.6440 & 7.0111 & 6.6562 \\
\hline & 3 & 28.190 & 26.781 & 24.024 & 21.623 & 19.481 & 17.527 & 16.686 \\
\hline & 4 & 28.404 & 26.895 & 24.098 & 21.886 & 20.088 & 18.391 & 17.459 \\
\hline & 5 & 51.618 & 48.878 & 43.787 & 39.732 & 36.403 & 33.262 & 31.575 \\
\hline \multirow{5}{*}{20} & 1 & 1.8070 & 1.7107 & 1.5332 & 1.3945 & 1.2839 & 1.1795 & 1.1199 \\
\hline & 2 & 11.196 & 10.600 & 9.4992 & 8.6383 & 7.9501 & 7.3014 & 6.9324 \\
\hline & 3 & 30.800 & 29.161 & 26.130 & 23.755 & 21.851 & 20.057 & 19.043 \\
\hline & 4 & 56.379 & 53.562 & 48.048 & 43.246 & 38.961 & 35.053 & 33.372 \\
\hline & 5 & 58.897 & 55.762 & 49.962 & 45.402 & 41.733 & 38.278 & 36.345 \\
\hline \multirow{5}{*}{30} & 1 & 1.8089 & 1.7126 & 1.5348 & 1.3960 & 1.2853 & 1.1809 & 1.1212 \\
\hline & 2 & 11.278 & 10.678 & 9.5691 & 8.7027 & 8.0112 & 7.3594 & 6.9876 \\
\hline & 3 & 31.325 & 29.657 & 26.576 & 24.165 & 22.239 & 20.425 & 19.394 \\
\hline & 4 & 60.681 & 57.449 & 51.475 & 46.795 & 43.049 & 39.525 & 37.532 \\
\hline & 5 & 84.569 & 80.343 & 72.072 & 64.870 & 58.442 & 52.580 & 50.058 \\
\hline \multirow{5}{*}{100} & 1 & 1.8103 & 1.7139 & 1.5360 & 1.3971 & 1.2863 & 1.1819 & 1.1222 \\
\hline & 2 & 11.340 & 10.736 & 9.6211 & 8.7506 & 8.0566 & 7.4026 & 7.0287 \\
\hline & 3 & 31.728 & 30.037 & 26.917 & 24.480 & 22.537 & 20.709 & 19.664 \\
\hline & 4 & 62.105 & 58.795 & 52.684 & 47.908 & 44.103 & 40.527 & 38.485 \\
\hline & 5 & 102.52 & 97.053 & 86.955 & 79.062 & 72.776 & 66.879 & 63.517 \\
\hline
\end{tabular}




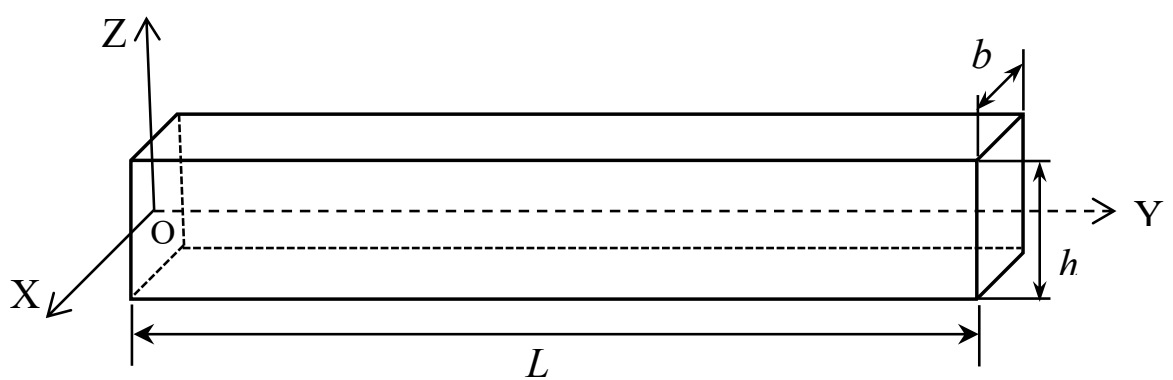

Fig. 1. The co-ordinate system and notation for a FGB 


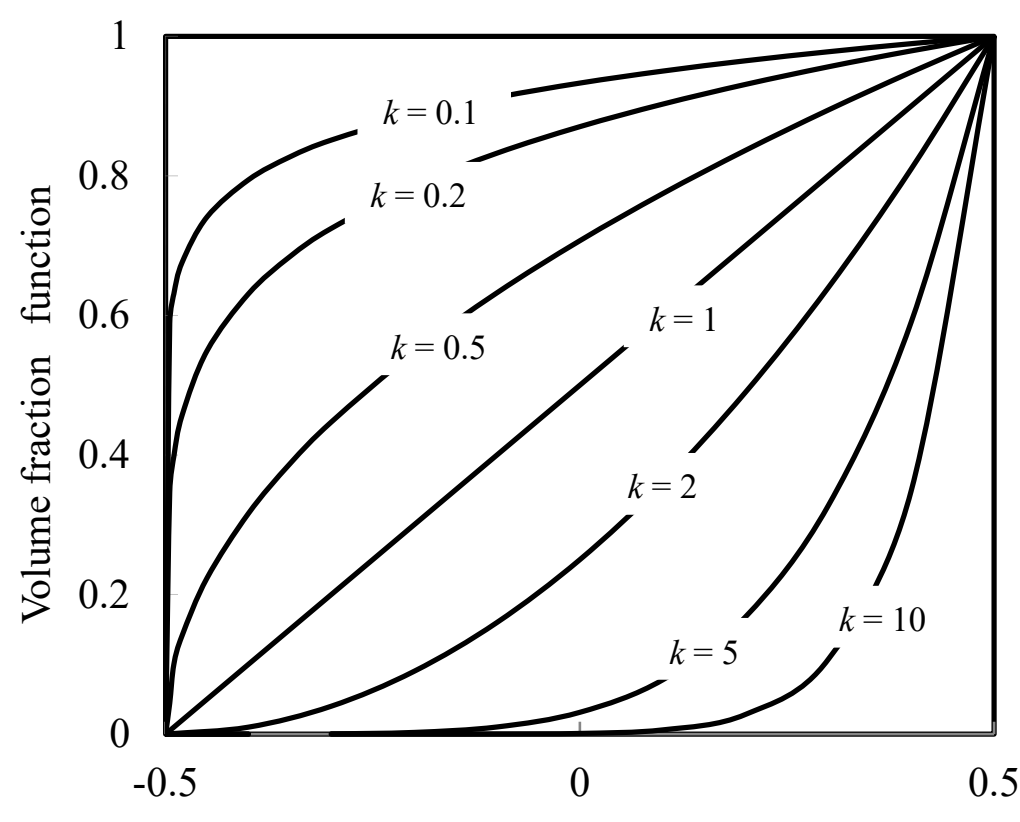

Thickness coordinate $\mathrm{z} / \mathrm{h}$

Fig. 2. Variation of volume fraction through the beam thickness in terms of $k$ 


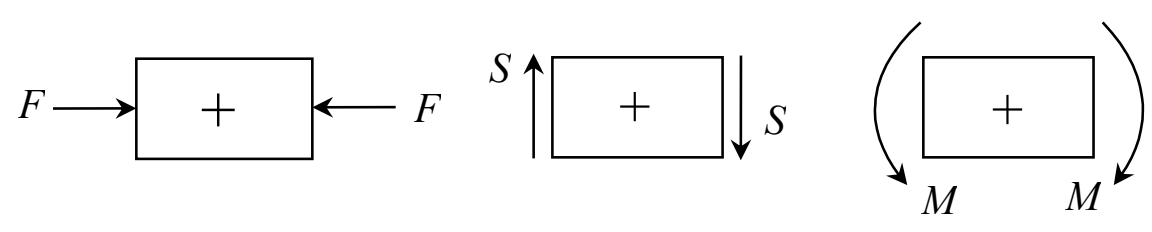

Fig. 3. Sign convention for positive axial force, shear force and bending moment 


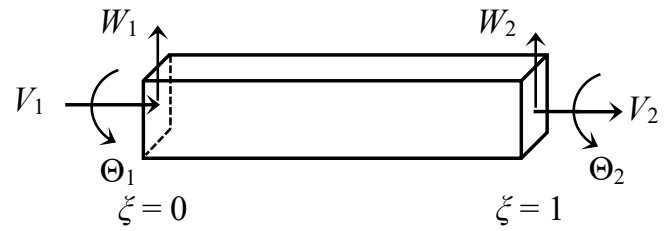

(a) Displacements

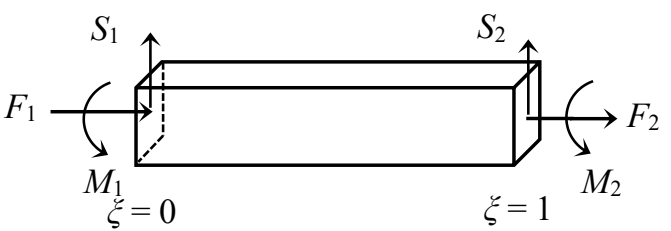

(b) Forces

Fig. 4. Boundary conditions for displacements (a) and forces (b) 


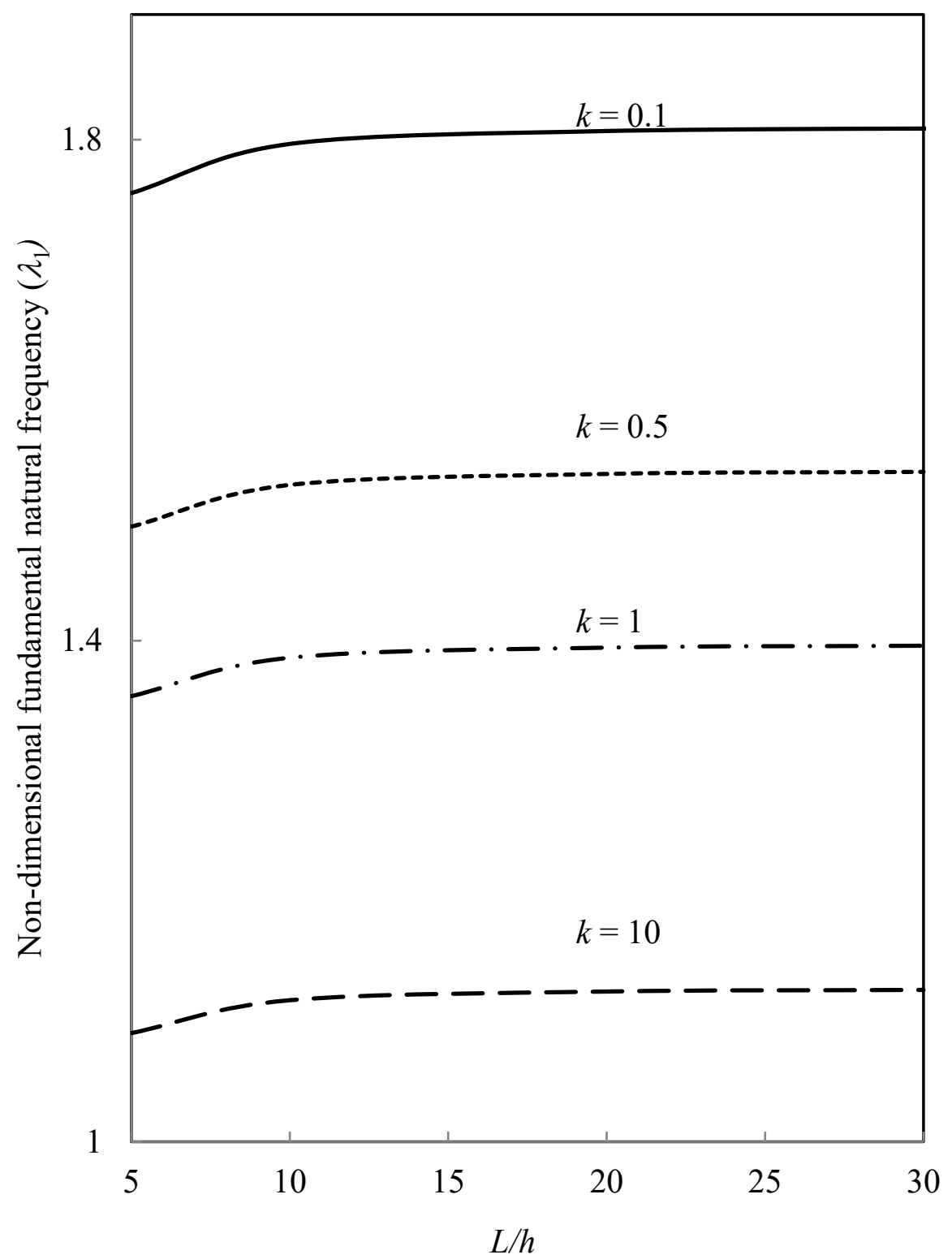

Fig. 5. The effect of the ratio $L / h$ on the fundamental natural frequency $\left(\lambda_{1}\right)$ of a cantilever FGB 


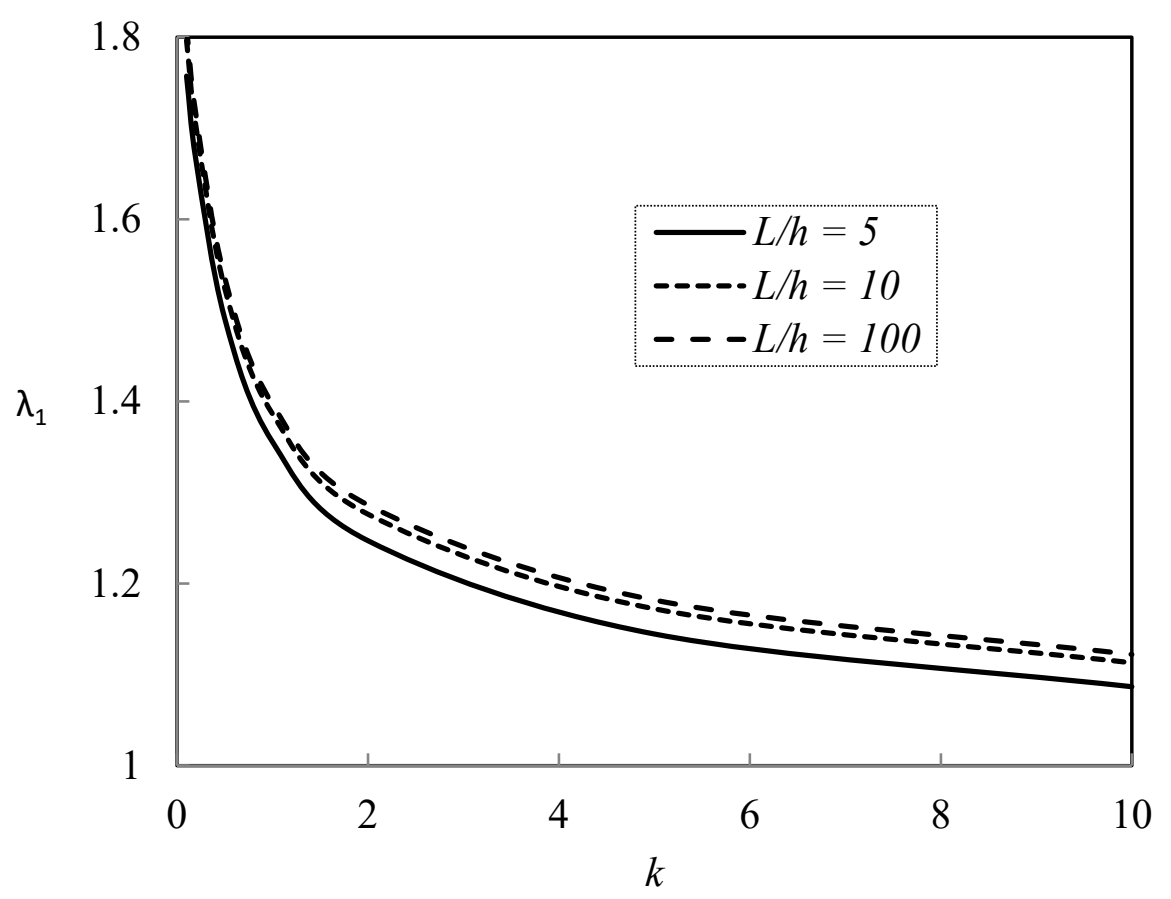

(a) Non-dimensional fundamental natural frequency $\left(\lambda_{1}\right)$

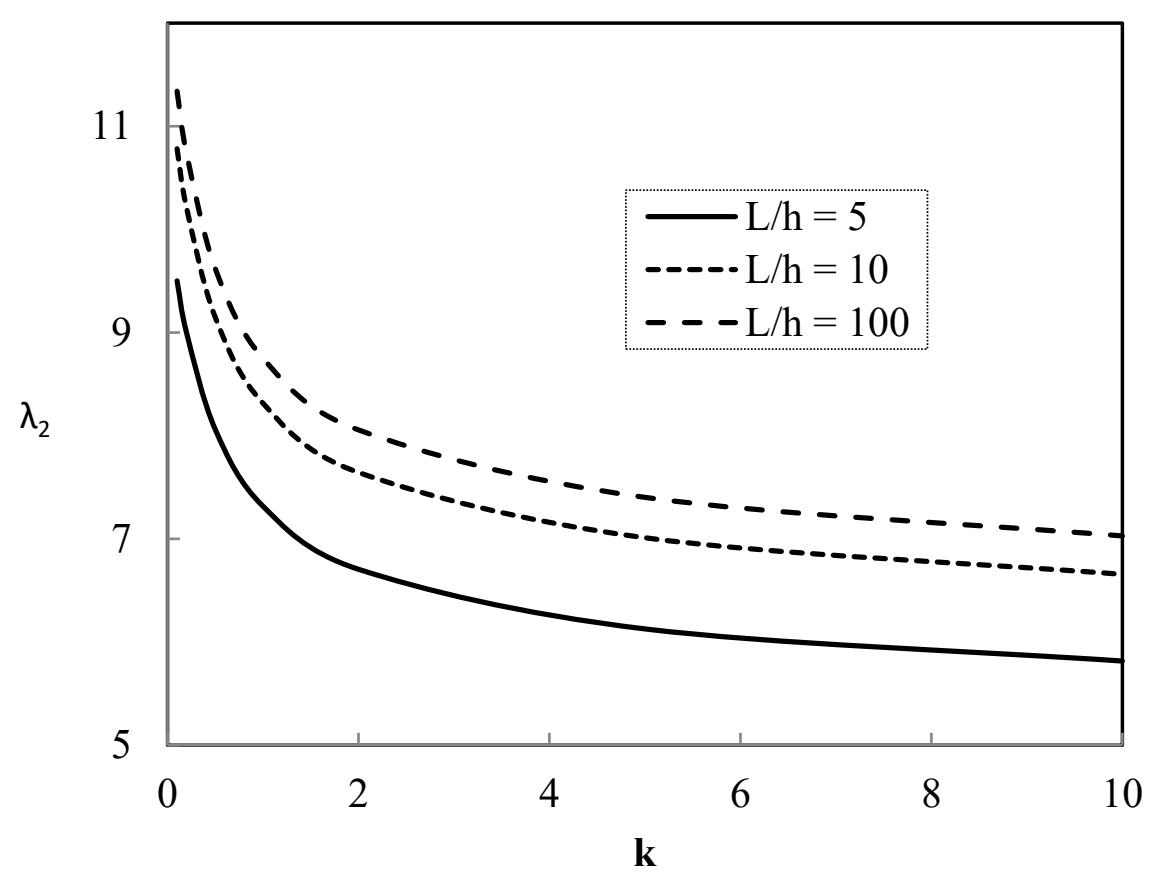

(b) The second non-dimensional natural frequency $\left(\lambda_{2}\right)$ 


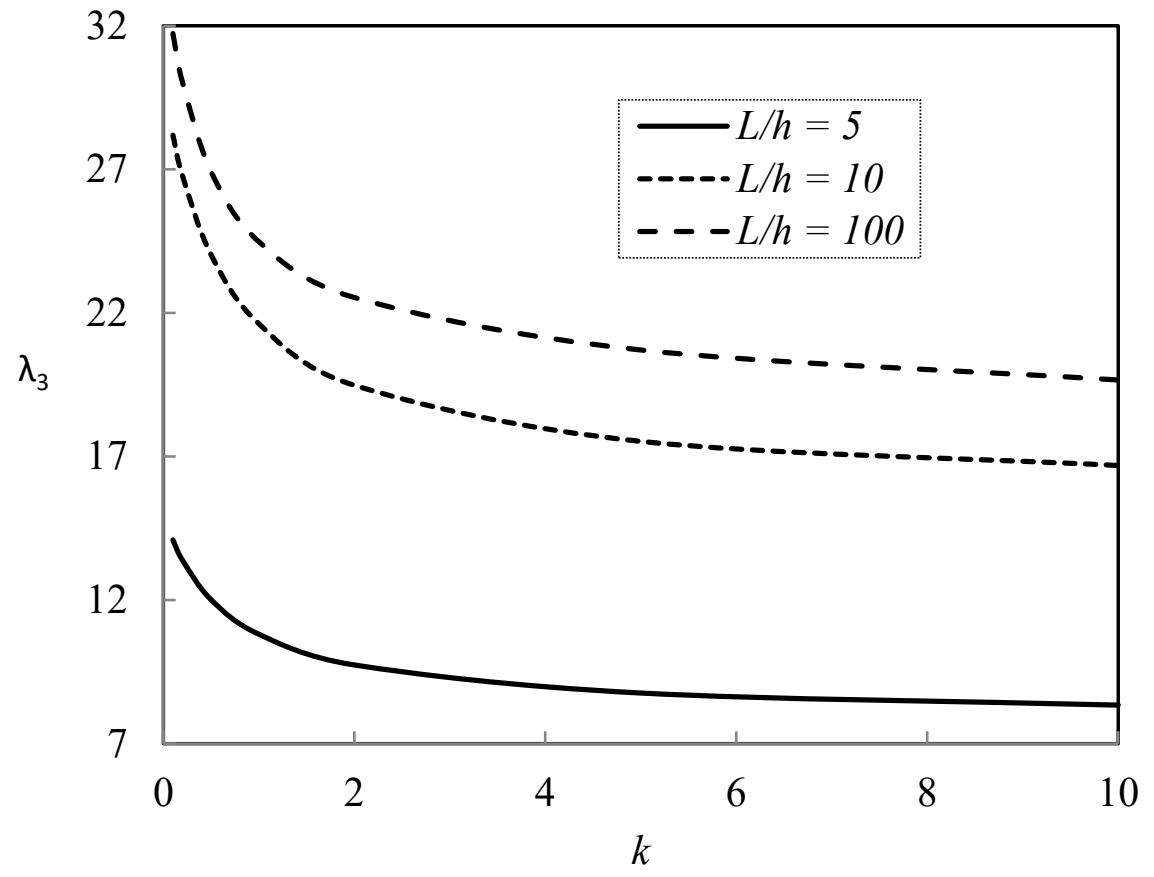

(c) The third non-dimensional natural frequency $\left(\lambda_{3}\right)$

Fig. 6. The effect of $k$ on the first three natural frequencies of a cantilever FGB 


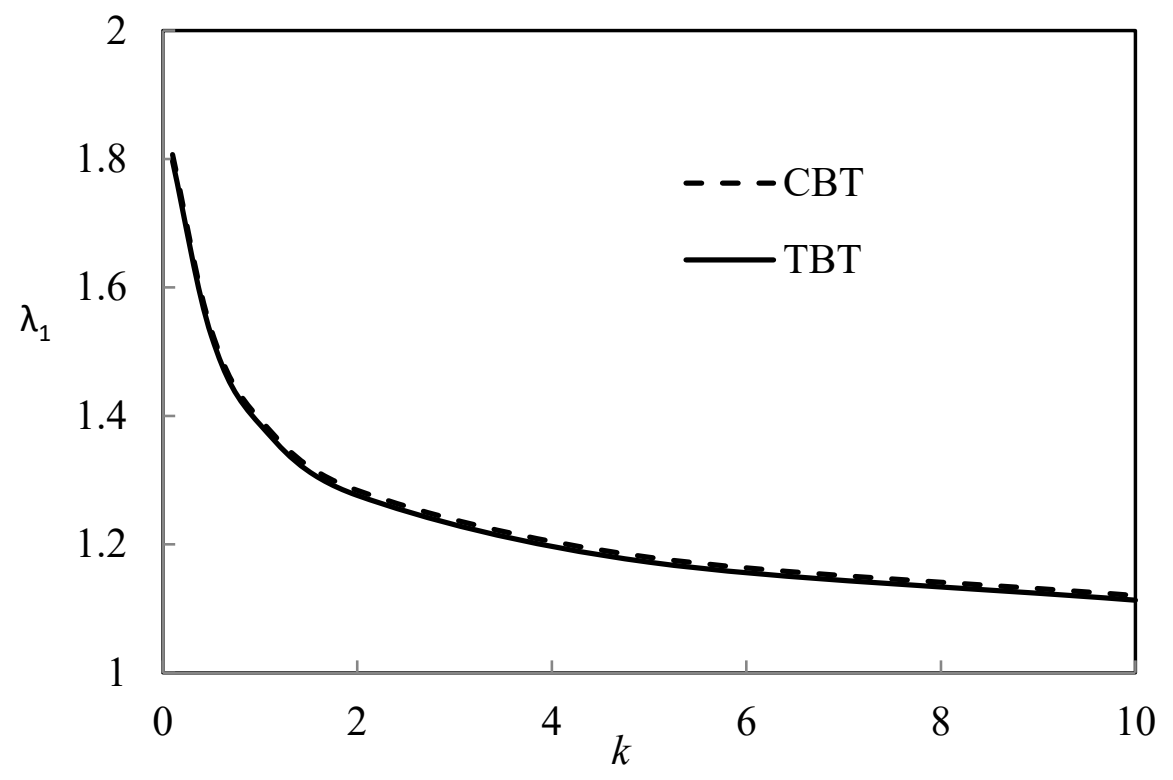

(a) Non-dimensional fundamental natural frequency $\left(\lambda_{1}\right)$

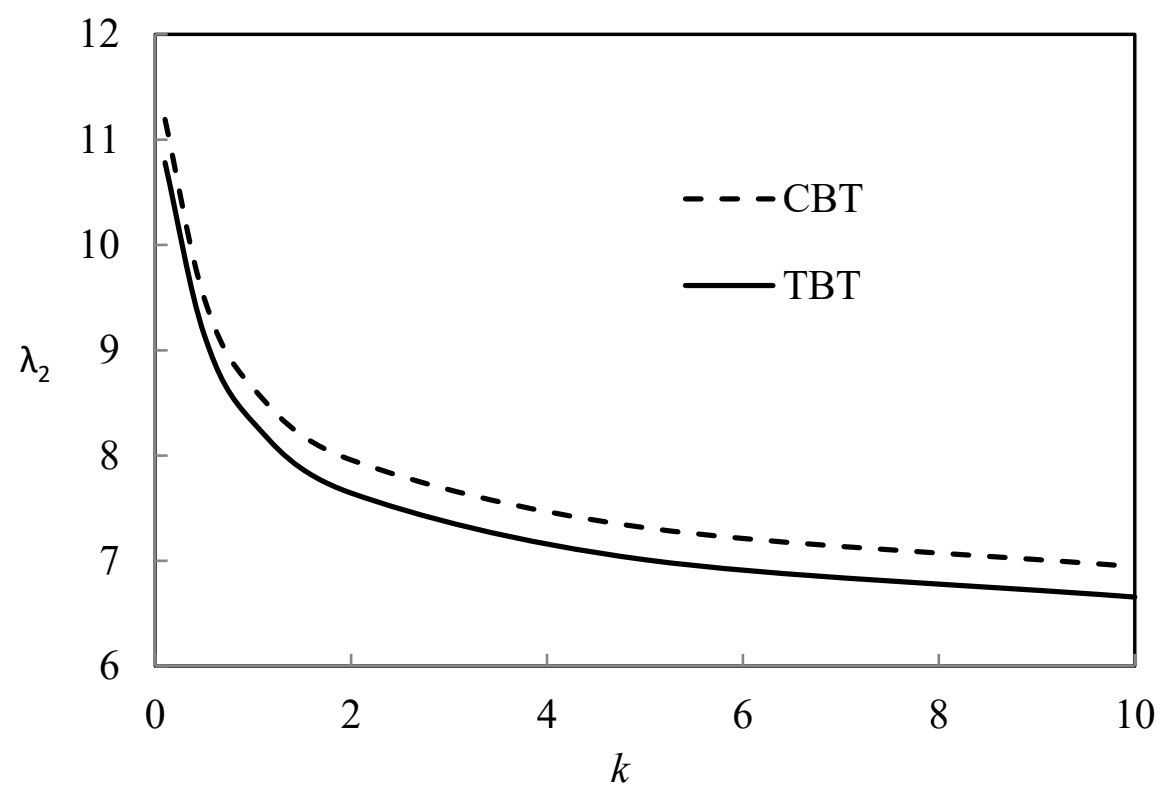

(b) The second non-dimensional natural frequency $\left(\lambda_{2}\right)$

Fig. 7. Variation of the first two natural frequencies of a cantilever FGB for $L / h=$ 10 using the CBT and TBT 


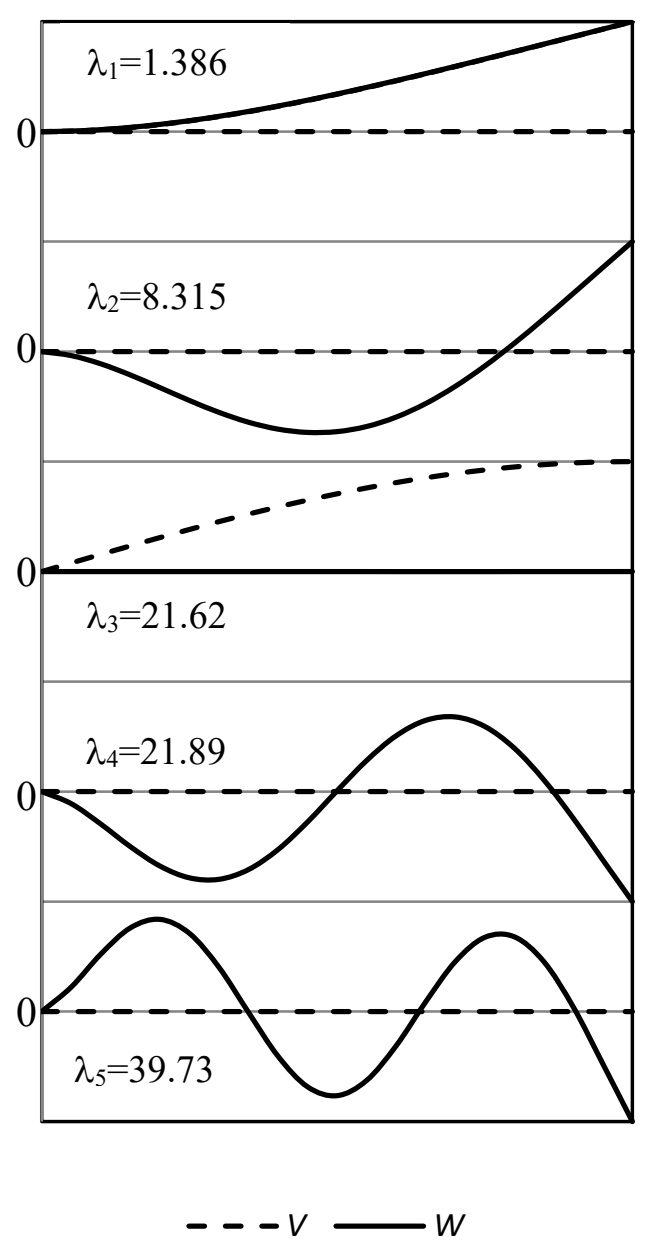

(a) $L / h=10$

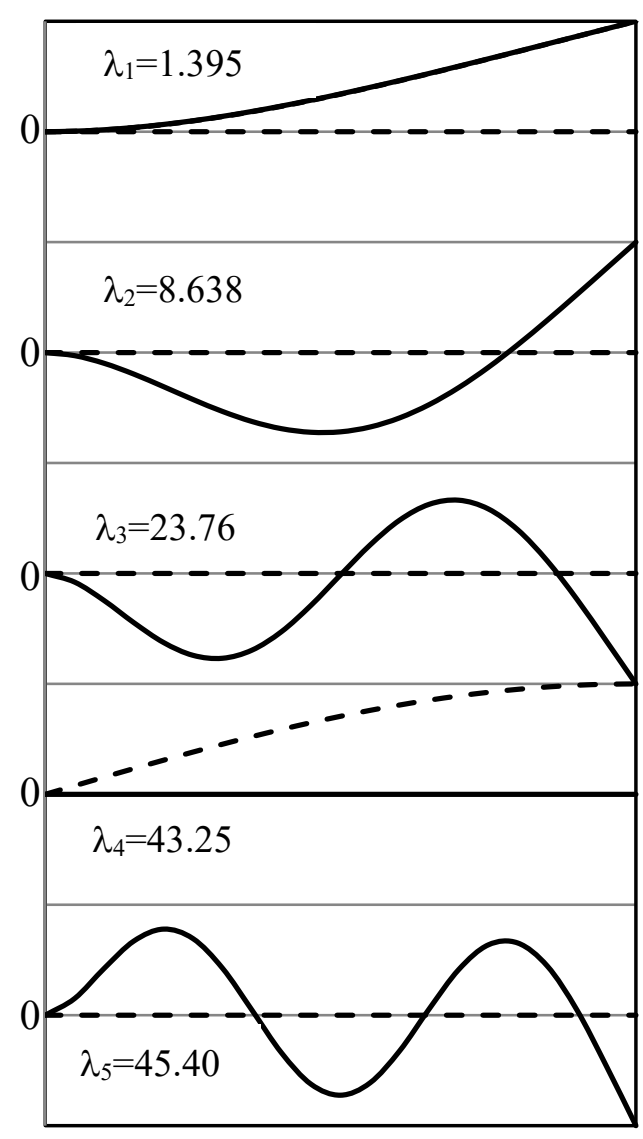

$---v \longrightarrow w$

(b) $L / h=20$

Fig. 8. The first five mode shapes of a cantilever FGB when $k=1$ for $L / h=10$ and 20 